\title{
АСТРОФИЗИКА
}

\author{
Э. В. Серга
}

\section{К ТЕОРИИ ФИЗИЧЕСКОГО ВАКУУМА: РАСПРОСТРАНЕНИЕ ЭЛЕКТРОМАГНИТНЫХ И ГРАВИТАЦИОННЫХ ВОЛН}

\begin{abstract}
Аннотация. Предметом настоящего исследования является космический (физический) вакуум какматериальная среда. Задачей исследования является поиск оснований для разработки непротиворечивого научного объяснения всей совокупности наблюдаемых вакуумных эффектов в физике микромира и макромира - единой теории вакуума. Представленная в статье теория вакуума является развитием воззрений на вакуум как материальную среду, сформировавшихся в трудах И. Ньютона, М. Фарадея, Д.К. Максвелла, Г.Р. Герца, с учетом результатов новейших научных исследований. Ключевыми предпосылками работы являются представления о симметрии гравитационных взаимодействий как физической реальности, а также о единстве теории вакуума в физике микромира и в физике космоса. Для решения поставленных задач использованы общенаучные методы и приемы исследования (обобщение, анализ, синтез), методы формальной логики, гипотетико-дедуктивный метод, моделирование. Особое внимание в работе уделено объяснению механизма распространения электромагнитных и гравитационных волн в вакууме. При разработке теоретической модели вакуума и объяснения с её использованием этих процессов в качестве исходной идеи был принят эффект рождения в вакууме пар «частица-античастица». Теоретически определённые спектры электромагнитных волн в различных диапазонах согласуются с наблюдаемыми значениями. Дано новое теоретическое определение возможной скорости гравитации, которая, по расчетам, составила порядка 10 в 18 степени скорости света.
\end{abstract}

Ключевые слова. Физический вакуум, Теории эфира, Электромагнитная волна, Гравитационная волна, Скорость гравитации, Антигравитация, Квантовая жидкость, Вакуумный эффект, Вакуумная пара, Комптоновская длина волны.

\begin{abstract}
The research subject is the space (physical) vacuum as a material medium. The research task is search for the grounds to the development of a consistent scientific explanation of the set of observed vacuum effects in microphysics and macrophysics - a unified vacuum theory. The presented vacuum theory is the continuation of the views of vacuum as a material medium formed in the works by I. Newton, M. Faraday, J.C. Maxwell and H.R. Hertz with account for the recent scientific research. The key premises of the study are the ideas about the symmetry of gravitational interactions as a physical reality and about the unity of the vacuum theory in microphysics and cosmophysics. To solve the research tasks, the author uses general scientific methods and methodologies (generalization, analysis, synthesis), the methods of formal logic, hypothetico-deductive method and modeling. Special attention is given to the explanation of the mechanism of transmission of electromagnetic and gravitational waves in vacuum. To develop the theoretical model of vacuum and explain its application using these processes, the author accepts the effect of occurrence of "particle-antiparticle" pairs in vacuum as a root idea. Theoretically determined spectra of electromagnetic waves in various diapasons conform with the observed values. The author formulates the new theoretical definition of possible gravitation speed which, according to the calculations, is about $10^{18}$ the speed of light.
\end{abstract}

Keywords: Vacuum effect, Quantum fluid, Anti-gravity, Speed of gravity, Gravitational wave, Electromagnetic wave, Aether theories, Physical vacuum, Vacuum pair, Compton wavelength. 


\section{Исследования космоса 3(4) • 2017}

\section{Введение}

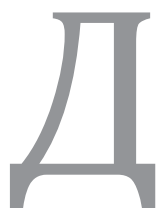
анная работа является развитием предложенной автором теории вакуума, позволяющей непротиворечиво объяснить сочетание в этом физическом явлении свойств пустого пространства, квантовой жидкости, а также плотной упругой среды, в которой распространяются электромагнитные и гравитационные волны, а тела могут совершать движение при отсутствии заметного сопротивления [1].

Согласно предлагаемой теории, вакуум состоит из вакуумных пар (ВП): «электрон-позитрон» и «протон-антипротон» (обозначения $<e^{-} e^{+}>$ и $<p^{+} p^{-}>$). ВП может быть устойчивой системой, если сила притяжения электрических зарядов уравновешена силой отталкивания гравитационных масс частицы и античастицы. Из условия равенства сил притяжения электрических зарядов и отталкивания гравитационных масс было получено расстояние между гравитационными полюсами $\ell_{g}$ :

$$
\ell_{g}=h / e c \cdot(\varepsilon G)^{1 / 2},
$$

где $h$ - постоянная Планка, $e$ - элементарный заряд, $c-$ скорость света, $\varepsilon=4 \pi \varepsilon_{0}, \varepsilon_{0}-$ электрическая постоянная, $G$ - гравитационная постоянная.

Под полюсами понимаются точки, в которых приложены равнодействующие сил притяжения электрических зарядов и отталкивания гравитационных масс ВП. Величина $\ell_{g}$ представляет собой гравитационный аналог комптоновской длины волны и составляет $1.2 \cdot 10^{-33}$ м. Она является одной и той же для ВП $<e^{-} e^{+}>$и $<p^{+} p^{-}>$, а также пар из двух одинаковых элементарных частиц (античастиц). Поэтому её можно рассматривать как фундаментальную постоянную (фундаментальную длину). ВП имеют целочисленный спин, нулевые значения электрического заряда и гравитационной массы, но не равную нулю инертную массу. Ранее [1] было показано, что состав и структура вакуума позволяют объяснить следующие наблюдаемые вакуумные эффекты в космосе:

- проявление сил инерции;

- круговые формы планетных орбит;
- $\quad$ аномальные смещения перигелиев планет.

Задача данного исследования состоит в объяснении с использованием предлагаемой теории механизма распространения в вакууме электромагнитных и гравитационных волн. Основное внимание было уделено анализу следующих вакуумных эффектов, известных в квантовой теории поля (КТП) и физике конденсированных сред (ФКС):

- Рождение и исчезновение в вакууме пар «частица-античастица»;

- Поляризация вакуума;

- Существование квантовых жидкостей, которые в основном невозбуждённом состоянии обладают свойством сверхтекучести, но обладают упругостью.

Вакуумные пары $<e^{-} e^{+}>$и $<p^{+} p^{-}>$рассматриваются как переносчики электромагнитных и гравитационных взаимодействий.

\section{1. Концептуальные подходы к проблеме распространения электромагнитных и гравитационных волн в вакууме}

Теория вакуума, претендующая на роль единой, должна объяснить, помимо прочего, свойства вакуума как среды, в которой распространяются электромагнитные и гравитационные волны. В части электромагнитных волн имеются опытные данные, которые можно использовать для оценки правильности предлагаемой теории. В части гравитационных волн таких данных нет. Нет также единого мнения и в части различных оценок скорости гравитации. В данной работе автором получены новые результаты, касающиеся оценки скорости гравитации.

Используемая в настоящей работе теория вакуума содержит три основные составляющие: 1) результаты обобщения и систематизации воззрений классиков науки на «вселенский эфир» как материальную среду, заполняющую мировое пространство; 2) новые данные о физическом вакууме, полученные благодаря современным возможностям науки; 3) концепция гравитационной симметрии.

1.1. Результаты обобщения и систематизации воззрений классиков науки на «вселенский эфир»

Логические обоснования «теории эфира» как среды, заполняющей мировое пространс- 
тво, были заложены в XVII-XIX веках в трудах И. Ньютона, Д.К. Максвелла, М. Фарадея, Г.Р. Герца и других выдающихся учёных.

Так, Ньютон полагал, что существует среда, через которую передаются взаимодействия между телами. Фарадей ввёл понятие поля и выдвинул идею о передаче электромагнитных взаимодействий посредством поля. Максвелл, развивая взгляды Фарадея, допустил существование среды, в которой распространяются электромагнитные волны. Поле рассматривается как возбуждённое состояние эфира и наделяется теми же характеристиками, что и вещество - энергией, массой, импульсом. То есть поле возникает как развитие идеи эфира, утверждая принцип близкодействия, и отвергая представление о мировом пространстве как пустоте. Концепция эфира как материальной среды получила дальнейшее развитие в механике Герца. Он приписывал частицам эфира свойства инертной материи [2]. Тем самым устраняется противоречие между появлением силы инерции в эфире и третьим законом механики Ньютона.

Однако уровень знаний, как и исследовательские возможности тех времен не позволяли объяснить сочетание в эфире свойств пустого пространства и плотной упругой среды. Согласно исследованиям П.-С. Лапласа, движение планет происходит в полном соответствии с законом Ньютона [3]. Следовательно, если эфир существует, то, согласно наблюдениям, он не влияет на движение небесных тел. Вместе с тем, от эфира как материальной среды нельзя отказаться потому, что в нём распространяется свет, представляющий собой электромагнитные волны. Эти противоречивые свойства эфира, несовместимые с представлениями о других известных средах, не позволили выдающимся учёным прошлого построить логически последовательную и замкнутую [4] теорию эфира.

\section{2. Новые данные о физическом вакууме}

Новые данные о физическом вакууме - это, прежде всего, вакуумные эффекты квантовой теории поля (КТП) и представления о вакууме как сверхтекучей квантовой жидкости в физике конденсированных сред (ФКС). В КТП известны вакуумные эффекты, которые характеризуют вакуум как среду, обладающую специфическими свойствами, отличающими её как от пустоты, так и обычных изученных сред. Это рождение в вакууме пар «частица-античастица», поляризация вакуума, сдвиг уровней энергии в атоме водорода (так называемый «лэмбовский сдвиг», названный в честь У. Лэмба (Lamb, Willis, 19132008) - одного из авторов открытия).

В 1938 г. П.Л. Капица открыл сверхтекучесть жидкого гелия. Это открытие положило начало новому научному направлению - физике конденсированных сред. В ФКС появились новые субстанции. Среди них есть жидкости, которые при определённой температуре испытывают фазовый переход в сверхтекучее состояние. Одно из проявлений сверхтекучести состоит в отсутствии вязкости: такая жидкость при нулевой температуре (т.е. находясь в основном невозбуждённом состоянии) может не оказывать сопротивления движущимся в ней телам, но обладает упругостью. Новые свойства этих жидкостей напоминают свойства эфира, а именно, сочетание противоречивых свойств пустоты и плотной упругой среды. Согласно представлениям ФКС, вакуум можно рассматривать как квантовую жидкость, лабораторным аналогом которой является жидкий гелий в сверхтекучем состоянии [5].

Таким образом, новые знания, которыми располагает современная наука, позволяет разрешить парадокс: сочетание в вакууме (эфире) противоречивых свойств - плотной упругой среды и пустого пространства. Свойство плотной упругой среды проявляется в распространении в вакууме электромагнитных и гравитационных волн, а наблюдаемое свойство пустого пространства проявляется в движении планет без сопротивления вакуума как среды. Новые знания позволяют возродить концепцию вселенского эфира, от которого физики отказались в начале $\mathrm{XX}$ века после появления специальной теории относительности, в которой А. Эйнштейн упразднил эфир. Вот что писал, например, известный физик-теоретик Г.Е. Воловик: «Если бы Ньютон знал о существовании подобных жидкостей, он смог бы разрешить сформулированный им парадокс, наделив эфир свойством сверхтекучести» $[5$, с. 90]. 


\section{Исследования космоса 3(4) • 2017}

1.3. Концепция гравитационной симметрии

Проблема гравитационной симметрии давно привлекала внимание учёных. Ещё в XIX веке ряд учёных пришёл к выводу, что мир, в котором гравитационные силы являются только силами тяготения, не может быть устойчивым и, следовательно, существовать во времени. Поэтому существование отрицательных гравитационных масс серьёзно рассматривалось как решение трудностей, когда ньютоновский закон тяготения был применён к Вселенной в целом. Исследования по этой проблеме были обобщены М. Джеммером (Jammer, Max, 1915-2010) [6]. Тот факт, что мы не наблюдаем взаимного отталкивания масс, может быть объяснён правдоподобным допущением, что тела с отрицательными гравитационными массами были оттолкнуты телами с положительными гравитационными массами, преобладающими в нашей области пространства, на расстояния, недоступные для нашего опыта [6, с. 137]. Ф. Энгельс в работе «Диалектика природы» писал: «Истинная теория материи должна отвести отталкиванию такое же важное место, как и притяжению, и что теория материи, основанная только на тяготении, ложна, недостаточна, половинчата» [7, с. 210-211]. Возможность существования отрицательных гравитационных масс допускал также Э. Шредингер (Schrödinger, Erwin, 1881-1661) [8].

Следует отметить, что законы взаимодействия гравитационных масс и электрических зарядов (закон Ньютона и закон Кулона) математически тождественны. В этих законах силы притяжения и отталкивания равноправны. Но инертная масса может быть только положительной, как это следует из второго закона механики Ньютона. Один из аргументов против антигравитации состоит в том, что она противоречит принципу эквивалентности инертной и гравитационной масс в общей теории относительности (ОТО). Однако неубедительность данного аргумента проявляется, в частности, в том, что этот принцип не помешал Эйнштейну ввести в обобщённые уравнения ОТО так называемый космологический член. Как отмечает В.Л. Гинзбург, физический смысл введения этого члена на классическом языке означает наличие сил отталкивания, действующих помимо сил тяготения [9]. По-видимому, убедительным способом разрешить спор между противниками и сторонниками гравитационной симметрии может стать эксперимент [1]. Представляется, что последовательная теория вакуума может быть построена лишь в случае признания гравитационной симметрии как факта физической реальности.

\section{0 методе исследования}

Ключевым методом настоящего исследования является формальная логика. Как известно, существует ряд причин, которые могут направить исследователя по ложному пути. Первая - это гипотезы, не имеющие достаточно надёжного подтверждения опытом. Вторая - произвольное определение некоторых ненаблюдаемых величин, которые не следуют из физических экспериментов. Третья причина состоит в том, что физическую проблему часто пытаются решить, как чисто математическую проблему. При этом добиваются согласования теории с опытными данными, оставляя вне поля зрения некоторые факторы, от которых зависит конечный результат.

Кроме указанных причин на выбор метода исследования и оценку правильности полученного результата нередко большое влияние оказывают субъективные факторы. Именно для того, чтобы свести к минимуму влияние субъективных факторов, следует использовать правила формальной логики.

В настоящей работе используется аксиоматический метод логического вывода, основы которого заложены в трудах классиков науки Д. Гильберта, Г. Вейля, А. Пуанкаре [10-12]. При этом были использованы правила логического вывода, которые в обобщённом и доступном изложении приведены в книге Ханса Фрейденталя (Freudenthal, Hans) «Язык логики» [13].

Суть авторского метода заключается в следующем. Теория (или её отдельный фрагмент) рассматривается как сложное высказывание, состоящее из простых высказываний, объединённых логическими связками. Сложное высказывания считается истинным, если составляющие его простые высказывания являются истинными. 
При этом используется процедура, которая называется выполнением высказывания. Она состоит в оценке истинности простых высказываний, при которых сложное высказывание будет истинным. Оценка истинности теории как сложного высказывания осуществляется на основе сравнения соответствия предсказаний теории с опытными данными.

Первоочередная цель исследования состоит в формулировке системы аксиом, которые используются для решения поставленной задачи. При этом использованы факты (события), между которыми были установлены причинно-следственные связи. При достаточном количестве таких верных утверждений из них можно вывести все нужные утверждения, которые подтверждаются опытом.

Согласно правилам формальной логики, умозаключения должны выводиться только из фактов, не допуская при этом противоречий. Каждое простое высказывание можно рассматривать как утверждение (следствие). В дальнейшем отдельные факты теоретически обобщаются в единую логически последовательную замкнутую систему. Построение цепи взаимосвязанных логических умозаключений должно предшествовать применению математики. При этом не обязательно использовать символы и обозначения языка логики, но следует строго следовать логическим правилам.

Как уже отмечалось, в качестве основополагающей идеи был принят вакуумный эффект рождения в вакууме пары «частица-античастица». Этот же эффект был использован в качестве основополагающей идеи в работах по теории вакуума ряда других авторов [см., например, 14, 15].

\section{3. Исходные положения, которые были использованы для объяснения механизма распространения электромагнитных и гравитационных волн}

Основополагающим положением является факт рождения в вакууме пар «частица-античастица» (вакуумных пар). Эти пары рассматриваем в качестве структурных элементов вакуума, определяющих его свойства как системы, состоящей из этих пар.
3.1. Рождение пары «частица-античастица».

Описание эффекта. Фотон, обладающий достаточной энергией, может вызвать появление в вакууме пары «частица-античастица». Компоненты пары непродолжительное время вращаются относительно общего геометрического центра, потом исчезают, испустив фотон с энергией, равной энергии поглощённого фотона.

\section{Следствия:}

1) вакуум состоит из пар «частица-античастица»: пар < $e^{-} e^{+}>$и $\left(p^{+} p^{-}>\right.$;

2) ВП в возбуждённом (наблюдаемом) состоянии представляют собой квантово-механические системы, подобные атому водорода;

3) ВП могут поглощать и излучать кванты электромагнитной энергии;

Вывод: для описания механизма распространения электромагнитных волн может быть использована боровская теория атома водорода.

4) спектры электромагнитных волн определяются разницей энергий переходов ВП из одного состояния в другое, разрешённые условиями квантования.

\section{2. Поляризация вакуума.}

Описание эффекта. Сторонний электрический заряд притягивает полярные (по отношению к нему) заряды вакуумных пар, что вызывает его частичную экранировку [16, с. 496].

Следствие: ВП в невозбуждённом (ненаблюдаемом) состоянии представляют собой электрические диполи.

\section{4. Электромагнитные волны}

Вакуумные пары $<e^{-} e^{+}>$и $<p^{+} p^{-}>$является частицами вакуума как среды, в которой распространяются электромагнитные волны. В возбуждённом состоянии ВП представляет собой систему по своей структуре подобную атому водорода. Отличие состоит в том, что компоненты пары вращаются относительно общего центра, а в атоме водорода электрон вращается относительно неподвижного протона. Поэтому для вывода основных соотношений использовалась боровская теория атома водорода. Тогда, согласно первому постулату Бора, электрон и 


\section{Исследования космоса 3(4) • 2017}

позитрон могут вращаться относительно геометрического центра пары, не излучая, только по определённым орбитам, разрешённым условием квантования. При этом наименьший орбитальный момент $L_{e}$ каждой из частиц пары равен $1 / 2 \hbar$. Тогда суммарный орбитальный момент должен быть равен 0 или ћ в зависимости от ориентации спинов каждой частицы.

Энергетическим уровням пар соответствуют квантовые числа $n$. Переход пары с одного уровня на другой связан с поглощением или испусканием фотона с энергией, равной разности энергий уровней пары в двух различных состояниях.

Применительно к паре $\left.<e^{-} e^{+}\right\rangle$, условие квантования можно сформулировать так: суммарный момент количества движения электрона и позитрона, движущихся относительно геометрического центра пары, кратен величине $\hbar$ :

$$
L=m_{e} r v=n \hbar, n=1,2,3 \ldots
$$

Это означает, что на орбите ВП должно быть целое число волн. Полагаем, что наименьшей возможной длиной волны ВП является комптоновская длина волны электрона $\lambda_{e}=h / m_{e} c\left(\lambda_{e}=\right.$ $\left.=2.424 \cdot 10^{-12} \mathrm{M}\right)$, которая соответствует энергии ВП с квантовым числом $n=1$. Тогда минимальный радиус орбиты $r_{\min }$ сквантовым числом $n=1$ будет равен $\lambda_{e} / 2 \pi$, а расстояние между электрическими полюсами $\ell_{1}$ будетравно диаметру орбиты, т.е. $\ell_{1}=$ $\lambda_{e} / \pi$. Тогда для состояния ВП с квантовым числом $n$ радиус её орбиты $r_{n}$ определяется как:

$$
r_{n}=n \lambda_{e} / 2 \pi
$$

Каждому разрешенному состоянию ВП соответствует определенная энергия, равная сумме потенциальной $U$ и кинетической $W$ энергий электрона и позитрона. Величина потенциальной энергии $U$ для ВП с квантовым числом $n$ определяется так:

$$
U=e^{2} / \varepsilon \ell_{n^{\prime}}\left(\ell_{n}=2 r_{n}\right)
$$

где $\varepsilon=4 \pi \varepsilon_{0}, \varepsilon_{\text {o }}$ - электрическая постоянная.

В нашем случае можно использовать теорему вириала. Согласно этой теореме, для системы вза- имодействующих по закону Кулона заряженных частиц кинетическая энергия $W$ равна половине потенциальной энергии $U$ с обратным знаком [19, c. 77-78; 20, с. 34-36]. Тогда полная энергия системы $E_{n}$ равна половине потенциальной энергии. Она определяется по формуле:

$$
E_{n}=1 / 2 e^{2} / \varepsilon \ell_{n^{*}}
$$

Если в формуле (4) использовать выражения для постоянной тонкой структуры $\alpha=e^{2} / \varepsilon \hbar c$ и энергии массы покоя $E^{\circ}=m_{e} c^{2}$, то оно приводится к виду:

$$
E_{n}=1 / 4 \cdot 1 / n \cdot E^{\circ} \alpha,
$$

Тогда энергии разрешенных квантовых переходов ВП из состояния с квантовым числом $n$ в состояние с квантовым числом $m$ определяются по формуле:

$$
\Delta E_{m n}=E_{m}-E_{n}=-1 / 4 E^{\circ} \alpha \cdot(1 / m-1 / n) .
$$

Обозначим $\Phi_{e}=1 / 4 \alpha E^{\circ}, K_{m n}=(1 / n-1 / m)$ и тогда получим:

$$
\Delta E_{m n}=\Phi_{e} \cdot K_{m n}
$$

Частота и длина волны поглощаемого или излучаемого фотона при переходе системы из одного состояния в другое:

$$
v_{m n}=\Delta E_{m n} / h, \lambda_{m n}=c / v_{m n}
$$

Полученные соотношения для пар $<e^{-} e^{+}>$ справедливы и для пар $<p^{+} p->$. В таблице 1 приведены диапазоны длин электромагнитных волн, определенных теоретически и наблюдаемых. Как следует из данных таблицы 1 , теоретически определённые значения длин волн согласуются с наблюдаемыми значениями. Это подтверждает правильность теории и позволяет объяснить спектры электромагнитных волн в вакууме. При этом полагаем, что квантовые переходы происходят преимущественно между ближайшими состояниями ВП. 
Диапазоны электромагнитных волн в вакууме

\begin{tabular}{|c|c|c|c|}
\hline \multirow{2}{*}{ Тип излучения } & \multicolumn{2}{|c|}{ Длины волн, $\mathbf{2}$} & \multirow{2}{*}{$\begin{array}{c}\text { Тип ВП } \\
\text { (квантовые числа } \mathrm{n} \text { ) } \\
(\mathbf{m}=\mathrm{n}+1)\end{array}$} \\
\cline { 2 - 3 } & Наблюдаемые* & Расчетные & $<p^{+} p^{-}>(1-3)$ \\
\hline ү-излучение & $<10^{-11}$ & $(1,4-8,7) \cdot 10^{-12}$ & $<p^{+} p^{-}>(4-17)$ \\
Рентген. излучение & $10^{-11}-10^{-8}$ & $1,5 \cdot 10^{-11}-2,2 \cdot 10^{-10}$ & $<e^{-} e^{+}>(1-2)$ \\
\hline УФ-излучение & $10^{-8}-4 \cdot 10^{-7}$ & $2,7 \cdot 10^{-9}-8,0 \cdot 10^{-8}$ & $<e^{-} e^{+}>(3-17)$ \\
\hline Видимое излучение & $4 \cdot 10^{-7}-7,4 \cdot 10^{-7}$ & $4,1 \cdot 10^{-8}-4,1 \cdot 10^{-7}$ & $<e^{-} e^{+}>(17-23)$ \\
\hline
\end{tabular}

Источник: [16, с. 178].

Полученные соотношения (6-7) справедливы для определения энергетических переходов вакуумных пар, находящихся в возбуждённом состоянии. Энергия, необходимая для перевода вакуумных пар из невозбуждённого (основного) состояния $(n=0)$ на первый возбуждённый уровень $(n=1)$ определяется так:

$$
\Delta E_{01}=E_{0}-E_{1}=2 E^{\circ}-1 / 4 E^{\circ} \alpha \sim 2 E^{\circ}
$$

Для пар $<e^{-} e^{+}>\Delta E_{01} \sim 1.02$ Мэв, для пар $<p^{+} p^{-}>$ $\Delta E_{01} \sim 1.87$ Гэв.

\section{5. Гравитационные волны}

Можно дать следующие общие соображения о механизме гравитационных взаимодействий с учётом полученных автором результатов. Обмен квантами гравитационной энергии происходит в результате их излучения и поглощения атомными ядрами небесных тел. Вакуумные пары являются переносчиками гравитационных взаимодействий. Они поглощают и излучают кванты гравитационной энергии на всём пути их движения от одного тела к другому. Отсюда следует, что частицы вакуума и частицы атомных ядер небесных тел должны иметь некоторые общие характеристики как источники и приёмники квантов гравитационной энергии. Такими характеристиками являются: расстояния между гравитационными полюсами и инертные массы частиц вещества и частиц вакуума.
Основная масса вещества вакуума находится в парах протон-антипротон, а вещества небесных тел, как полагаем, - в ядерных парах (спаренных протонах). Существование спаренных протонов предполагаем по аналогии с существованием спаренныхэлектронов (куперовских пар), известных в теории сверхпроводимости $[16,21]$.

Излучение и поглощение квантов гравитационной энергии происходит в результате смещения гравитационных полюсов на расстояние, разрешённое условиями квантования. Как было показано ранее, это расстояние, определяемое как гравитационный аналог комптоновской длины волны, составляет: $\ell_{g}=1.2 \cdot 10^{-33} \mathrm{M}$.

Электрические и гравитационные полюса ядерных и вакуумных пар совершают энергетически разрешённые квантовые переходы, определённые различными условиями квантования. В выражении, содержащем первый постулат Бора, состояния, разрешённые условиями квантования, зависят от инертной массы. А инертная масса является только положительной как для частиц, так и античастиц.

Таким образом, вакуумные пары являются переносчиками гравитационных взаимодействий подобно тому, как они являются переносчиками электромагнитных взаимодействий. Но здесь есть особенность. Электроны в веществе небесных тел составляют ничтожную долю их гравитационной массы (а также инертной массы). Поэтому переносчиками гравитационных взаимодействий являются пары $<p^{+} p^{-}>$, а излу- 


\section{Исследования космоса 3(4) • 2017}

чателями и приёмниками гравитационных волн являются ядерные пары $<p^{+} p^{+}>$, а также, как полагаем, пары $<p^{*} p>$ в объектах из антивещества.

В настоящее время нет надёжных данных о спектрах гравитационных волн, которые можно было бы использоватьдля проверки излагаемой теории. Одной из проблем остаётся вопрос о скорости распространения гравитации в вакууме $v_{g}$. Как известно, Эйнштейн принял без каких-либо экспериментальных доказательств, что гравитация распространяется со скоростью света $c$ :

$$
v_{g}=c
$$

Однако нет оснований считать равенство (10) справедливым, пока эксперименты не дадут ответа. Эту проблему рассматривал Л. Бриллюен (Brillouin, Leon, 1889-1969) [22]. Он отмечает, что нет надёжных экспериментальных данных, подтверждающих равенство (10).

Нижний предел скорости гравитации был установлен П.-С. Лапласом в XVIII веке. Исследовав причины векового ускорения Луны, он сделал вывод о том, что скорость гравитации $v_{g}$ не менее чем в 50 млн. раз превышает скорость света с [3]. Здесь важна не точность полученной Лапласом величины, а обоснование того, что скорость гравитации не равна скорости света и на много порядков превышает её. Если учесть, что весь опыт расчётов положения планет в небесной механике базируется на статической формуле Ньютона, подразумевающей бесконечную скорость гравитации, то следует считать оценку Лапласа более верной, чем постулированное Эйнштейном равенство скоростей света и гравитации.

Полученные в настоящей работе результаты позволяют дать собственную оценку скорости гравитации на основе знания состава и характеристик вакуумных пар как вероятных переносчиков гравитационных волн. При этом, как представляется, скорость гравитации зависит от тех же фундаментальных постоянных, которые связывают скорость света с комптоновской длиной волны частицы, т.е. постоянной планка $h$ и инертной массы частицы $m$. Тогда для определения скорости гравитации $v_{g}$ используем зависимость между скоростью и длиной волны в соотношении Л. де Бройля, заменив комптоновскую длину волны протона на значение её гравитационного аналога $\ell_{g=} 1.2 \cdot 10^{-33} \mathrm{M}$ :

$$
v_{g}=h / m_{p} \ell_{g}
$$

Подставив значения входящих в это выражение величин, получим:

$$
v_{g}=3,3 \cdot 10^{26} \text { м/с или } 1.1 \cdot 10^{18} c .
$$

Полученное значение $v_{g}$ можно считать правильным при условии, что постоянная Планка $h$ является универсальной как для электромагнитных, так и гравитационных взаимодействий. Основанием для такого предположения является размерность величины $h$, определяемая в Дж.с. Она не зависит от фундаментальных величин, характеризующих гравитационные и электромагнитные взаимодействия (в частности, констант связи в законах Ньютона и Кулона).

\section{Заключение}

В статье получила дальнейшее развитие теоретическая модель физического (космического) вакуума как формы материи [1]. В частности, рассмотрены приложения данной модели к объяснению механизма распространения электромагнитных и гравитационных волн в вакууме. Согласно предлагаемой модели, вакуум состоит из вакуумных пар «электрон-позитрон» и «протон-антипротон». Вакуумные пары рассматривались как квантово-механические системы, подобные по своей структуре атому водорода. Поэтому для определения спектров энергетических переходов вакуумных пар использовалась боровская теория атома водорода. Теоретически определённые спектры электромагнитных волн согласуются с наблюдаемыми спектрами.

По аналогии с распространением электромагнитных волн дано объяснение механизма распространения гравитационных волн. Согласно предлагаемой теории, источниками и приёмниками гравитационных волн являются спаренные протоны (ядерные пары) в составе атомных ядер, а переносчиками гравитационных волн являются вакуумные пары «протон-антипротон». Исходя из положения об универсальности постоянной Планка для электромагнитных и гравитационных взаимодействий, дана оценка скорости гравитации, которая составила $10^{18}$ скорости света. 


\section{Библиография}

1. Серга Э. В. Физический вакуум как форма материи: новый взгляд на структуру и свойства // Исследования космоса. 2017. №2. С. 85-100.

2. Герц Г. Принципы механики, изложенные в новой связи / Серия «Классики науки». М.: Изд. АН CCCP, 1959. C. 386.

3. Laplace P. S. Mecanique celeste, 4, livre X. Paris, 1805.

4. Гейзенберг В. Шаги за горизонт. - М.: Прогресс, 1987. С. 178-189.

5. Воловик Г. Е. От эфира Ньютона к вакууму современной физики конденсированных сред // Ньютон и философские проблемы ХХ века. - М.: Наука, 1991. С. 88-98.

6. Энгельс Ф. Диалектика природы. - М.: Политиздат, 1969.

7. Джеммер М. Понятие массы в классической и современной физике. Пер. с англ. - М.: Прогресс, 1967. - 256 c.

8. Schrödinger E. Über das Losungssystem der allgemein kovarianten Gravitatiosglechungen // Physikalische Zeitschrift. 1918. Vol. 19. P. 20-22.

9. Гинзбург В. Л. О теории относительности. Сб. статей. - М.: Наука, 1979. С. 94.

10. Гильберт Д., Бернайс П. Основания математики. Теория доказательств. - М.: Наука, 1982. -652 c.

11. Вейль Г. Математическое мышление. Пер. с англ. и нем. - М.: Наука, 1989. - 400 с.

12. Пуанкаре А. 0 науке. Пер. с франц. - М.: Наука, 1983. - 560 с.

13. Фрейденталь Х. Язык логики. Пер. с англ. - М.: Наука, 1969. - 136 с.

14. Рыков А. В. Вакуум и вещество во Вселенной. - М.: Изд. «Рестарт». 289 с.

15. Герловин И.Л. Основы теорий всех взаимодействий в веществе. - Л.: Энергоатомиздат, Ленинградское отд., 1990. - 432 с.

16. Физика микромира: маленькая энциклопедия / гл. ред. чл.-корр. АН СССР Д. В. Ширков. - М.: Советская энциклопедия, 1980.

17. Физическая энциклопедия. Т. 1. / гл. ред. акад. АН СССР А. М. Прохоров. - М.: Советская энциклопедия, 1988.

18. Большая Российская энциклопедия. Т. 4. Научное издание «Большая Российская энциклопедия. - М., 2006.

19. Физический энциклопедический словарь / гл. ред. акад. АН СССР А.М. Прохоров. - М.: Советская энциклопедия, 1983.

20. Ландау Л.Д., Лифшиц Е. М. Теоретическая физика. Т.1. Механика. - М.: Наука, 1965. - 204 с.

21. Cooper L. N. Bound electron pairs in a degenerate Fermi gas // Physical Review. 1956. Vol. 104. №4. P. 1189-1190.

22. Бриллюен Л. Новый взгляд на теорию относительности. Пер. с англ. - М.: Мир, 1972. - 143 с.

\section{References (transliterated)}

1. Serga E. V. Fizicheskii vakuum kak forma materii: novyi vzglyad na strukturu i svoistva // Issledovaniya kosmosa. 2017. №2. S. 85-100.

2. Gerts G. Printsipy mekhaniki, izlozhennye v novoi svyazi / Seriya «Klassiki nauki». M.: Izd. AN SSSR, 1959. S. 386.

3. Laplase P. S. Mecanique celeste, 4, livre X. Paris, 1805.

4. Geizenberg V. Shagi za gorizont. - M.: Progress, 1987. S. 178- 189.

5. Volovik G. E. Ot efira N'yutona k vakuumu sovremennoi fiziki kondensirovannykh sred // N'yuton i filosofskie problemy KhKh veka. - M.: Nauka, 1991. S. 88-98.

6. Engel's F. Dialektika prirody. - M.: Politizdat, 1969.

7. Dzhemmer M. Ponyatie massy v klassicheskoi i sovremennoi fizike. Per. s angl. - M.: Progress, 1967. $-256 \mathrm{~s}$. 


\section{Исследования космоса 3(4) • 2017}

8. Schrödinger E. Über das Losungssystem der allgemein kovarianten Gravitatiosglechungen // Physikalische Zeitschrift. 1918. Vol. 19. P. 20-22.

9. Ginzburg V. L. O teorii otnositel'nosti. Sb. statei. - M.: Nauka, 1979. S. 94.

10. Gil'bert D., Bernais P. Osnovaniya matematiki. Teoriya dokazatel'stv. - M.: Nauka, 1982. - $652 \mathrm{~s}$.

11. Veil' G. Matematicheskoe myshlenie. Per. s angl. i nem. - M.: Nauka, 1989. - 400 s.

12. Puankare A. O nauke. Per. s frants. - M.: Nauka, 1983. - 560 s.

13. Freidental' Kh. Yazyk logiki. Per. s angl. - M.: Nauka, 1969. - $136 \mathrm{s.}$

14. Rykov A. V. Vakuum i veshchestvo vo Vselennoi. - M.: Izd. «Restart». - 289 s.

15. Gerlovin I.L. Osnovy teorii vsekh vzaimodeistvii v veshchestve. - L.: Energoatomizdat, Leningradskoe otd., 1990. $-432 \mathrm{~s}$.

16. Fizika mikromira: malen'kaya entsiklopediya / gl. red. chl.-korr. AN SSSR D. V. Shirkov. - M.: Sovetskaya entsiklopediya, 1980.

17. Fizicheskaya entsiklopediya. T. 1. / gl. red. akad. AN SSSR A. M. Prokhorov. - M.: Sovetskaya entsiklopediya, 1988.

18. Bol'shaya Rossiiskaya entsiklopediya. T. 4. Nauchnoe izdanie «Bol'shaya Rossiiskaya entsiklopediya. - M., 2006.

19. Fizicheskii entsiklopedicheskii slovar' / gl. red. akad. AN SSSR A.M. Prokhorov. - M.: Sovetskaya entsiklopediya, 1983.

20. Landau L.D., Lifshits E. M. Teoreticheskaya fizika. T.1. Mekhanika. - M.: Nauka, 1965. - 204 s.

21. Cooper L. N. Bound electron pairs in a degenerate Fermi gas // Physical Review. 1956. Vol. 104. №4. P. 1189-1190.

22. Brillyuen L. Novyi vzglyad na teoriyu otnositel'nosti. Per. s angl. - M.: Mir, 1972. - 143 s. 